PROCEEDINGS OF THE

AMERICAN MATHEMATICAL SOCIETY

Volume 133, Number 7, Pages 2119-2129

S 0002-9939(05)07776-2

Article electronically published on February 15, 2005

\title{
ON RESIDUALITIES IN THE SET OF MARKOV OPERATORS ON $\mathcal{C}_{1}$
}

\author{
WOJCIECH BARTOSZEK AND BEATA KUNA
}

(Communicated by Joseph A. Ball)

\begin{abstract}
We show that the set of those Markov operators on the Schatten class $\mathcal{C}_{1}$ such that $\lim _{n \rightarrow \infty}\left\|P^{n}-Q\right\|=0$, where $Q$ is one-dimensional projection, is norm open and dense. If we require that the limit projections must be on strictly positive states, then such operators $P$ form a norm dense $G_{\delta}$. Surprisingly, for the strong operator topology operators the situation is quite the opposite.
\end{abstract}

\section{INTRODUCTION}

The Baire category theorem has a long history in ergodic theory. The first proof (see [11] for all the details) that there are nonmixing but weakly mixing transformations was based on this theorem (other constructive methods followed but were more complicated). Some time later Baire methods were successfully applied (compare [7], [8], [18]) in the ergodic theory of Markov operators defined on $L^{1}(\mu)$ (i.e. such that $P f \geq 0$ and $\int P f d \mu=\int f d \mu$ for all nonnegative $f$ ). Baire type considerations usually bring easy answers to existence problems (see for instance 4], [5, 12, 13, 14 for recent applications). Typical questions concern the size of a specific class of operators (ergodic, conservative, with convergent iterations). Similarly as in 13, we will show in a noncommutative environment that the answers depend heavily on the point of view, i.e. on the choice of topology. We shall see that the set of mixing operators is meager in the strong operator topology but it is residual in the norm topology (even if we require a very fast, exponential, rate of mixing in the operator norm).

We begin our paper by introducing Markov operators on the simplest noncommutative von Neumann algebra of all bounded operators on a separable Hilbert space. As was pointed out by the referee, this topic has recently attracted the attention of specialists and some of our results should hold true for general von Neumann algebras with separable preduals. The reader is referred to [1, [9] and 10. for details and further references.

Let $(\mathcal{H},\langle\cdot, \cdot\rangle)$ be a separable (infinite-dimensional) complex Hilbert space. As usual the norm is denoted by $\|\cdot\|$ and the Banach algebra of linear and bounded

Received by the editors May 16, 2003 and, in revised form, April 7, 2004.

2000 Mathematics Subject Classification. Primary 46L55, 47A35; Secondary 37A55, 47B60.

Key words and phrases. Schatten classes, Markov operator, mixing operator, residuality.

The authors thank the referee for pointing out a gap in the first version of the proof of Theorem 2.6.

(C)2005 American Mathematical Society Reverts to public domain 28 years from publication 
operators on $(\mathcal{H},\|\cdot\|)$ is denoted by $\mathcal{L}(\mathcal{H})$. Without confusion the operator norm in $\mathcal{L}(\mathcal{H})$ will be denoted by $\|\cdot\|$ too. This paper is devoted to linear operators acting on an ordered Banach space of trace-class operators on $\mathcal{H}$. Let us recall some standard concepts and notation from the theory of linear operators on Hilbert spaces. The reader is referred to any standard book on operators on Hilbert spaces (for instance [6], 15, 16], 17] or [20]). The adjoint operator to $A$ is denoted by $A^{*}$. An operator $A \in \mathcal{L}(\mathcal{H})$ is called hermitian if $A=A^{*}$, i.e. $\langle A x, y\rangle=\langle x, A y\rangle$ holds for all $x, y \in \mathcal{H}$. Equivalently, an operator $A$ is hermitian if $\langle A x, x\rangle \in \mathbb{R}$ for any $x \in \mathcal{H}$ (see [6]). Moreover, if $\langle A x, x\rangle \in[0, \infty)$ holds for all $x \in \mathcal{H}$, then we say that $A$ is positive. Clearly, positive operators on $\mathcal{H}$ form a cone in $\mathcal{L}(\mathcal{H})$, denoted by $\mathcal{L}(\mathcal{H})_{+}$. Each hermitian operator $A$ may be uniquely decomposed as $A=A_{+}-A_{-}$ (with $A_{+} A_{-}=A_{-} A_{+}=0$ ), where $A_{+}$and $A_{-}$are called respectively a positive and negative part of $A$. By $|A|$ we mean $A_{+}+A_{-}$. Obviously $|A| \in \mathcal{L}(\mathcal{H})_{+}$, and it is called a modulus of $A$. The modulus may be equivalently introduced as $|A|=\sqrt{A^{*} A}$ (see [6]). Given the cone, we introduce in $\mathcal{L}(\mathcal{H})$ a partial order by $A \leq B$ if and only if $B-A \in \mathcal{L}(\mathcal{H})_{+}$. It is well known that $\mathcal{L}(\mathcal{H})$ endowed with this order is not a (vector) lattice and it does not satisfy the so-called Riesz decomposition property (see [6]). A general bounded operator $A$ may be written as $A=B+i C=\left(B_{+}-B_{-}\right)+i\left(C_{+}-C_{-}\right)$, where both $B, C$ are hermitian. An important role in Hilbert theory is played by compact operators. Let us recall that $A$ is compact if $A\left(x_{n}\right)$ has a (norm) convergent subsequence for each bounded sequence $x_{n} \in \mathcal{H}$ (or equivalently (see [17]) when $A$ is a norm operator limit of finitedimensional operators). Compact operators play an important role in Hilbert space theory. They form a (closed) ideal in $\mathcal{L}(\mathcal{H})$, which is denoted in our paper by $\mathcal{C}_{0}$. We say that an operator $X \in \mathcal{L}(\mathcal{H})$ is trace-class if for each (some; see [17] for all the details) orthonormal basis $e_{1}, e_{2}, \cdots \in \mathcal{H}$ the series $\sum_{j=1}\left\langle|X| e_{j}, e_{j}\right\rangle<\infty$. The trace is defined as $\sum_{j=1}\left\langle X e_{j}, e_{j}\right\rangle$ and it is denoted by $\operatorname{tr}(X)$. Then the functional

$$
X \rightarrow \operatorname{tr}(|X|)=\|X\|_{1}
$$

defines (see [16, [17) a norm (stronger than the operator norm). The trace-class operators form a two-sided ideal in $\mathcal{L}(\mathcal{H})$, which is called the Schatten class 1 (see [17], 20]), and it is denoted by $\mathcal{C}_{1}$. The trace norm is complete on $\mathcal{C}_{1}$. It may be easily verified that whenever $\mathcal{H}$ is not finite dimensional, then $\mathcal{C}_{1}$ is not closed in the operator norm in $\mathcal{L}(\mathcal{H})$. It is well known (see [17]) that by the dual operation $\langle A, X\rangle=\operatorname{tr}(X A)$, where $A \in \mathcal{C}_{0}$ and $X \in \mathcal{C}_{1}$, the adjoint space to $\left(\mathcal{C}_{0},\|\cdot\|\right)$ may be identified with $\left(\mathcal{C}_{1},\|\cdot\|_{1}\right)$. Further, the dual space to $\left(\mathcal{C}_{1},\|\cdot\|_{1}\right)$ is $(\mathcal{L}(\mathcal{H}),\|\cdot\|)$ (denoted in this context as $\mathcal{C}_{\infty}$ ) with dual operation $\langle X, B\rangle=\operatorname{tr}(B X)$, where $B \in \mathcal{C}_{\infty}$ and $X \in \mathcal{C}_{1}$. In particular, $\mathcal{C}_{1}$ is not reflexive. The space $\mathcal{C}_{1}$ is commonly recognized as the noncommutative counterpart of $\ell^{1}$ space. Since the operators of finite rank are norm dense in $\mathcal{C}_{1}$, and the Hilbert space $\mathcal{H}$ is separable (by our assumption), thus $\mathcal{C}_{1}$ is separable too. The following additivity property (sometimes called the $(\mathrm{AL})$ condition when we deal with Banach lattices) of the norm $\|\cdot\|_{1}$ is preserved:

$$
\forall X_{1}, X_{2} \in \mathcal{C}_{1}\left(X_{1}, X_{2} \geq 0 \Rightarrow\left\|X_{1}+X_{2}\right\|_{1}=\left\|X_{1}\right\|_{1}+\left\|X_{2}\right\|_{1}\right) .
$$

A noncommutative analog of $\ell^{p}$ space, called the Schatten class $\mathcal{C}_{p}$, exists too, but it is not used in our paper. 
Definition 1.1. A positive operator $X$ from $\mathcal{C}_{1}$ is called a state if $\operatorname{tr}(X)=1$. The set of all states is denoted by S.

It is easy to verify that $\mathrm{S}$ is a convex and closed subset of $\mathcal{C}_{1}$ for the weak topology (hence for both operator and trace norms). By a direct inspection it can be shown that it is not closed for the weak* topology (if $\operatorname{dim} \mathcal{H}=\infty$ ).

Definition 1.2. A bounded linear operator $P: \mathcal{C}_{1} \rightarrow \mathcal{C}_{1}$ is said to be positive if $P\left(\mathcal{C}_{1+}\right) \subseteq \mathcal{C}_{1+}$. A positive operator $P$ is called Markov (markovian) if for every $X \in \mathcal{C}_{1+}$ we have $\|P(X)\|_{1}=\|X\|_{1}$ (equivalently we may say that $P(\mathrm{~S}) \subseteq \mathrm{S}$ ). The set of all markovian operators on $\mathcal{C}_{1}$ is denoted by $\mathcal{S}$.

There are several natural topologies used in studying the geometry of the set $\mathcal{S}$ (and its subsets). First of all we have a norm operator topology inherited from the Banach space $\mathcal{L}\left(\mathcal{C}_{1}, \mathcal{C}_{1}\right)$ of all bounded linear operators on $\mathcal{C}_{1}$. Again the norm in $\mathcal{L}\left(\mathcal{C}_{1}, \mathcal{C}_{1}\right)$ is denoted simply by $\|\cdot\|$. Additionally we have

(1) The strong operator topology (s.o.t.) is defined by the base sets

$$
\left\{P \in \mathcal{L}\left(\mathcal{C}_{1}, \mathcal{C}_{1}\right):\left\|P\left(X_{j}\right)-P_{0}\left(X_{j}\right)\right\|_{1}<\varepsilon, \quad j=1, \ldots, n\right\}, \quad n \in \mathbb{N},
$$
where $X_{1}, X_{2}, \ldots$ are dense in $\mathcal{C}_{1}$.

(2) The weak operator topology (w.o.t.) is defined by the base sets

$$
\left\{P \in \mathcal{L}\left(\mathcal{C}_{1}, \mathcal{C}_{1}\right):\left|\operatorname{tr}\left(\left(P\left(X_{j}\right)-P_{0}\left(X_{j}\right)\right) A_{j}\right)\right|<\varepsilon, \quad j=1, \ldots, n\right\}, \quad n \in \mathbb{N},
$$

where $X_{1}, X_{2}, \cdots \in \mathcal{C}_{1}$ and $A_{1}, A_{2}, \cdots \in \mathcal{L}(\mathcal{H})$ are dense.

(3) The weak* operator topology (w*.o.t.) is defined by the base sets

$$
\left\{P \in \mathcal{L}\left(\mathcal{C}_{1}, \mathcal{C}_{1}\right):\left|\operatorname{tr}\left(A_{j}\left(P\left(X_{j}\right)-P_{0}\left(X_{j}\right)\right)\right)\right|<\varepsilon, \quad j=1, \ldots, n\right\}, \quad n \in \mathbb{N},
$$

where $X_{1}, X_{2}, \cdots \in \mathcal{C}_{1}$ and $A_{1}, A_{2}, \cdots \in \mathcal{C}_{0}$ are dense.

The following result is obvious, so it is left without a proof. Namely:

Lemma 1.3. The set $\mathcal{S}$ of all markovian operators on $\mathcal{C}_{1}$ is a convex and w.o.t. closed subsemigroup of $\mathcal{L}\left(\mathcal{C}_{1}, \mathcal{C}_{1}\right)$. However it is not closed for the $w^{*}$.o.t.

Now we give a few examples of Markov operators.

Example 1.4. Let $U$ be a unitary operator on $\mathcal{H}$. Define $P(X)=U^{*} X U$ and $Q(X)=U X U^{*}$. Clearly both $P$ and $Q$ are markovian. Moreover, they are invertible isometries of $\mathcal{C}_{1}$.

Example 1.5. Let $V$ be a linear contraction (onto) of $\mathcal{H}$ such that $V^{*}$ is isometric. Similarly as above we define $R(X)=V^{*} X V$. It is easy to check that $R$ is a markovian (noninvertible in general) operator on $\mathcal{C}_{1}$.

Example 1.6. It follows from the above lemma that any convex combination

$$
\sum_{j} \alpha_{j} P_{j}+\sum_{k} \beta_{k} Q_{k}+\sum_{l} \gamma_{l} R_{l}
$$

is markovian as long as $\alpha_{j}, \beta_{k}, \gamma_{l} \geq 0$ and $\sum_{j} \alpha_{j}+\sum_{k} \beta_{k}+\sum_{l} \gamma_{l}=1$. A slight modification gives

$$
\int P(s) d \nu(s) \in \mathcal{S}
$$

whenever all $P(s) \in \mathcal{S}$ and the integral over a probabilistic measure $\nu$ is properly defined. 


\section{NORM RESIDUALITY}

This paper is devoted to geometric properties of sets of operators $P \in \mathcal{S}$ such that $\lim _{n \rightarrow \infty} P^{n}$ exists and is rank one (i.e. $P$ is mixing). Of course we have different kinds of mixing depending on considered topologies. We begin with the strongest case, the norm mixing. Moreover, the convergence holds with an exponential rate. Even though the ideas for our first result come from [19] (see also [3] and [4]), for the completeness of the paper (and convenience of the reader) we have decided to include a detailed proof.

Lemma 2.1. Let $P$ be a Markov operator on $\mathcal{C}_{1}$. Then the following conditions are equivalent:

(i) there exist a one-dimensional projection $Q_{X_{*}} \in \mathcal{S}$ (i.e. $Q_{X_{*}}(X)=\operatorname{tr}(X) X_{*}$ for some $\left.X_{*} \in \mathrm{S}\right)$ and constants $C>0,0<a<1$ such that

$$
\left\|P^{n}-Q_{X_{*}}\right\|<C a^{n} \text { for } n \in \mathbb{N},
$$

(ii) there exists a one-dimensional projection $Q_{X_{*}} \in \mathcal{S}$ such that

$$
\lim _{n \rightarrow \infty}\left\|P^{n}-Q_{X_{*}}\right\|=0
$$

(iii) for each $\varepsilon>0$ there exists an index $n_{0}$ such that for all $X_{1}, X_{2} \in \mathrm{S}$ we have

$$
\left\|P^{n_{0}}\left(X_{1}\right)-P^{n_{0}}\left(X_{2}\right)\right\|_{1}<\varepsilon,
$$

(iv) there exists an index $n_{0}$ such that

$$
\lambda=\sup _{X_{1}, X_{2} \in \mathrm{S}}\left\|P^{n_{0}}\left(X_{1}\right)-P^{n_{0}}\left(X_{2}\right)\right\|_{1}<2 .
$$

Proof. We easily check that $\left\|Q_{X_{*}}(X)\right\|_{1}=\left\|(\operatorname{tr} X) X_{*}\right\|_{1}=(\operatorname{tr} X)\left\|X_{*}\right\|_{1}=1$ and $\left\langle Q_{X_{*}}(X) x, x\right\rangle=\left\langle(\operatorname{tr} X) X_{*} x, x\right\rangle=\operatorname{tr}(X)\left\langle X_{*} x, x\right\rangle=\left\langle X_{*} x, x\right\rangle \geq 0$ for all $x \in \mathcal{H}$ and $X \in \mathcal{C}_{1+}$. Therefore $Q_{X_{*}} \in \mathcal{S}$.

(i) $\Rightarrow$ (ii) Since $0 \leq\left\|P^{n}-Q_{X_{*}}\right\| \leq C a^{n}$ and $a<1$, thus the convergence $\lim _{n \rightarrow \infty}\left\|P^{n}-Q_{X_{*}}\right\|=0$ is obvious.

(ii) $\Rightarrow$ (iii) Given $\varepsilon>0$, let $\varepsilon_{1}=\frac{\varepsilon}{2}$. There exists $n_{0}$ such that for all $n \geq n_{0}$ we have $\left\|P^{n}-Q_{X_{*}}\right\|<\varepsilon_{1}$. Now, let $X_{1}, X_{2} \in \mathrm{S}$ be arbitrary. Then

$$
\begin{aligned}
& \left\|P^{n_{0}}\left(X_{1}\right)-P^{n_{0}}\left(X_{2}\right)\right\|_{1} \\
& =\left\|P^{n_{0}}\left(X_{1}\right)-Q_{X_{*}}\left(X_{1}\right)-P^{n_{0}}\left(X_{2}\right)+Q_{X_{*}}\left(X_{2}\right)+Q_{X_{*}}\left(X_{1}\right)-Q_{X_{*}}\left(X_{2}\right)\right\|_{1} \\
& \leq\left\|P^{n_{0}}\left(X_{1}\right)-Q_{X_{*}}\left(X_{1}\right)\right\|_{1}+\left\|P^{n_{0}}\left(X_{2}\right)-Q_{X_{*}}\left(X_{2}\right)\right\|_{1}+\left\|Q_{X_{*}}\left(X_{1}\right)-Q_{X_{*}}\left(X_{2}\right)\right\|_{1} \\
& <\frac{\varepsilon}{2}+\frac{\varepsilon}{2}=\varepsilon
\end{aligned}
$$

(iii) $\Rightarrow$ (iv) is obvious.

(iv) $\Rightarrow$ (i) In this part of our proof we repeat some arguments used in [3] and now adapted to the noncommutative case.

Let us note that for all states $X_{1}, X_{2} \in \mathrm{S}$ we have

$$
\operatorname{tr}\left(X_{1}-X_{2}\right)_{+}-\operatorname{tr}\left(X_{1}-X_{2}\right)_{-}=\operatorname{tr}\left(X_{1}-X_{2}\right)=\operatorname{tr}\left(X_{1}\right)-\operatorname{tr}\left(X_{2}\right)=0 .
$$

It follows from the above that

$$
\begin{aligned}
& \left\|X_{1}-X_{2}\right\|_{1}=\operatorname{tr}\left|X_{1}-X_{2}\right| \\
& =\operatorname{tr}\left(X_{1}-X_{2}\right)_{+}+\operatorname{tr}\left(X_{1}-X_{2}\right)_{-}=\operatorname{tr}\left|\left(X_{1}-X_{2}\right)_{+}\right|+\operatorname{tr}\left|\left(X_{1}-X_{2}\right)_{-}\right| \\
& =2 \operatorname{tr}\left|\left(X_{1}-X_{2}\right)_{+}\right|=2\left\|\left(X_{1}-X_{2}\right)_{+}\right\|_{1} .
\end{aligned}
$$


Because $P^{n_{0}}\left(X_{1}\right), P^{n_{0}}\left(X_{2}\right) \in \mathrm{S}$ we get

$$
\left\|P^{n_{0}}\left(X_{1}\right)-P^{n_{0}}\left(X_{2}\right)\right\|_{1}=2\left\|\left[P^{n_{0}}\left(X_{1}\right)-P^{n_{0}}\left(X_{2}\right)\right]_{+}\right\|_{1} .
$$

Therefore, $\left\|\left[P^{n_{0}}\left(X_{1}\right)-P^{n_{0}}\left(X_{2}\right)\right]_{+}\right\|_{1}=\frac{1}{2}\left\|P^{n_{0}}\left(X_{1}\right)-P^{n_{0}}\left(X_{2}\right)\right\|_{1}$ for all $X_{1}, X_{2} \in$ S. Since all iterates $P^{n}$ are contractions, thus

$$
\begin{aligned}
& \left\|P^{2 n_{0}}\left(X_{1}\right)-P^{2 n_{0}}\left(X_{2}\right)\right\|_{1}=\left\|P^{n_{0}}\left(P^{n_{0}}\left(X_{1}\right)\right)-P^{n_{0}}\left(P^{n_{0}}\left(X_{2}\right)\right)\right\|_{1} \\
& =\left\|P^{n_{0}}\left(P^{n_{0}}\left(X_{1}\right)-P^{n_{0}}\left(X_{2}\right)\right)\right\|_{1} \\
& =\left\|P^{n_{0}}\left\{\left[P^{n_{0}}\left(X_{1}\right)-P^{n_{0}}\left(X_{2}\right)\right]_{+}-\left[P^{n_{0}}\left(X_{1}\right)-P^{n_{0}}\left(X_{2}\right)\right]_{-}\right\}\right\|_{1} \\
& =\left\|\left[P^{n_{0}}\left(X_{1}\right)-P^{n_{0}}\left(X_{2}\right)\right]_{+}\right\|_{1} \cdot \| P^{n_{0}}\left(\frac{\left[P^{n_{0}}\left(X_{1}\right)-P^{n_{0}}\left(X_{2}\right)\right]_{+}}{\left\|\left[P^{n_{0}}\left(X_{1}\right)-P^{n_{0}}\left(X_{2}\right)\right]_{+}\right\|_{1}}\right) \\
& -P^{n_{0}}\left(\frac{\left[P^{n_{0}}\left(X_{1}\right)-P^{n_{0}}\left(X_{2}\right)\right]_{-}}{\left\|\left[P^{n_{0}}\left(X_{1}\right)-P^{n_{0}}\left(X_{2}\right)\right]_{-}\right\|_{1}}\right) \|_{1} \\
& \leq \lambda\left\|\left[P^{n_{0}}\left(X_{1}\right)-P^{n_{0}}\left(X_{2}\right)\right]_{+}\right\|_{1} \\
& \leq \frac{\lambda}{2}\left\|X_{1}-X_{2}\right\|_{1}=\beta\left\|X_{1}-X_{2}\right\|_{1},
\end{aligned}
$$

where by (iv) $\beta=\frac{\lambda}{2}<1$. We conclude that (so far only on $\mathrm{S}$ ) the mapping $P$ is eventually a strict contraction. Clearly $\mathrm{S}$ is a complete metric space, as it is a closed subset of a Banach space $\mathcal{C}_{1}$. Applying the Banach fixed point theorem, there exists a unique $P$-invariant state $X_{*} \in \mathrm{S}$, such that

$$
\lim _{n \rightarrow \infty}\left\|P^{n}(X)-X_{*}\right\|_{1}=0
$$

where $X \in \mathrm{S}$ is arbitrary. Let us take a one-dimensional Markov operator (projection) $Q_{X_{*}}(X)=\operatorname{tr}(X) X_{*}$. We note that $P Q_{X_{*}}=Q_{X_{*}} P=Q_{X_{*}}$. For an arbitrary $k \in \mathbb{N}$ the above method yields

$$
\begin{aligned}
\| P^{n_{0} k}\left(X_{1}\right) & -P^{n_{0} k}\left(X_{2}\right) \|_{1} \\
& \leq\left\|\left[P^{n_{0}}\left(X_{1}\right)-P^{n_{0}}\left(X_{2}\right)\right]_{+}\right\|\left\|_{1} \cdot\right\| P^{n_{0}(k-1)}\left(Y_{1}\right)-P^{n_{0}(k-1)}\left(Y_{2}\right) \|_{1} \\
& \leq \beta \cdot\left\|P^{n_{0}(k-1)}\left(Y_{1}\right)-P^{n_{0}(k-1)}\left(Y_{2}\right)\right\|_{1},
\end{aligned}
$$

where

$$
Y_{1}=\frac{\left[P^{n_{0}}\left(X_{1}\right)-P^{n_{0}}\left(X_{2}\right)\right]_{+}}{\left\|\left[P^{n_{0}}\left(X_{1}\right)-P^{n_{0}}\left(X_{2}\right)\right]_{+}\right\|_{1}} \quad \text { and } \quad Y_{2}=\frac{\left[P^{n_{0}}\left(X_{1}\right)-P^{n_{0}}\left(X_{2}\right)\right]_{-}}{\left\|\left[P^{n_{0}}\left(X_{1}\right)-P^{n_{0}}\left(X_{2}\right)\right]_{-}\right\|_{1}} .
$$

Substituting $X_{2}=X_{*}$ and iterating the above estimation we easily get

$$
\begin{aligned}
\left\|P^{n}\left(X_{1}\right)-Q_{X_{*}}\left(X_{1}\right)\right\|_{1} & =\left\|P^{n}\left(X_{1}\right)-X_{*}\right\|_{1} \\
& =\left\|P^{n}\left(X_{1}\right)-P^{n}\left(X_{*}\right)\right\|_{1} \leq C \alpha^{n},
\end{aligned}
$$

for all $X_{1} \in \mathrm{S}$, where $C=\frac{2}{\beta}$ and $\alpha=\beta^{1 / k_{0}}$. 
Finally let us consider general $Z \in \mathcal{C}_{1}$. Note that $Z=Z_{1}+i Z_{2}$ where $Z_{1}, Z_{2}$ are self-adjoint and $Z_{1}=Z_{1+}-Z_{1-}, Z_{2}=Z_{2+}-Z_{2-}$. Obviously $\left\|Z_{1}\right\|_{1}+\left\|Z_{2}\right\|_{1} \leq$ $2\|Z\|_{1}$. Now

$$
\begin{aligned}
& P^{n}(Z)=P^{n}\left(Z_{1+}\right)-P^{n}\left(Z_{1-}\right)+i P^{n}\left(Z_{2+}\right)-i P^{n}\left(Z_{2-}\right) \\
& =\left\|Z_{1+}\right\|_{1} P^{n}\left(\frac{Z_{1+}}{\left\|Z_{1+}\right\|_{1}}\right)-\left\|Z_{1-}\right\|_{1} P^{n}\left(\frac{Z_{1-}}{\left\|Z_{1-}\right\|_{1}}\right) \\
& +i\left\|Z_{2+}\right\|_{1} P^{n}\left(\frac{Z_{2+}}{\left\|Z_{2+}\right\|_{1}}\right)-i\left\|Z_{2-}\right\|_{1} P^{n}\left(\frac{Z_{2-}}{\left\|Z_{2-}\right\|_{1}}\right) .
\end{aligned}
$$

To obtain (i) we write

$$
\begin{aligned}
\| P^{n}(Z) & -Q_{X_{*}}(Z)\left\|_{1} \leq\right\| P^{n}\left(Z_{1+}\right)-Q_{X_{*}}\left(Z_{1+}\right)\left\|_{1}+\right\| P^{n}\left(Z_{1-}\right)-Q_{X_{*}}\left(Z_{1-}\right) \|_{1} \\
& +\left\|P^{n}\left(Z_{2+}\right)-Q_{X_{*}}\left(Z_{2+}\right)\right\|_{1}+\left\|P^{n}\left(Z_{2-}\right)-Q_{X_{*}}\left(Z_{2-}\right)\right\|_{1} \\
& =\left\|Z_{1+}\right\|_{1}\left\|P^{n}\left(\frac{Z_{1+}}{\left\|Z_{1+}\right\|_{1}}\right)-X_{*}\right\|_{1}+\left\|Z_{1-}\right\|_{1}\left\|P^{n}\left(\frac{Z_{1-}}{\left\|Z_{1-}\right\|_{1}}\right)-X_{*}\right\|_{1} \\
& +\left\|Z_{2+}\right\|_{1}\left\|P^{n}\left(\frac{Z_{2+}}{\left\|Z_{2+}\right\|_{1}}\right)-X_{*}\right\|_{1}+\left\|Z_{2-}\right\|_{1} P^{n}\left(\frac{Z_{2-}}{\left\|Z_{2-}\right\|_{1}}\right)-X_{*} \|_{1} \\
& \leq 2 C \alpha^{n}\|Z\|_{1} .
\end{aligned}
$$

Definition 2.2. We say that a Markov operator $P \in \mathcal{S}$ is norm mixing if one of the conditions of Lemma 2.1 is satisfied for some $n$ and some $\varepsilon<2$. The family of all norm mixing Markov operators is denoted by $\mathcal{S}_{n m}$.

Lemma 2.3. The set $\mathcal{S}_{n m}$ is a norm operator topology dense subset of $\mathcal{S}$.

Proof. Given a state $Y$, an arbitrary $P \in \mathcal{S}$, and $0<\varepsilon<1$, consider a convex combination

$$
P_{\varepsilon}(X)=(1-\varepsilon) P(X)+\varepsilon Q_{Y}(X) .
$$

By convexity $P_{\varepsilon} \in \mathcal{S}$. We will show that $P_{\varepsilon} \in \mathcal{S}_{n m}$. For any pair of states $X_{1}, X_{2}$ we have

$$
\begin{aligned}
& \left\|P_{\varepsilon}\left(X_{1}-X_{2}\right)\right\|_{1}=\left\|P_{\varepsilon}\left(X_{1}\right)-P_{\varepsilon}\left(X_{2}\right)\right\|_{1} \\
& =\left\|(1-\varepsilon) P\left(X_{1}\right)+\varepsilon\left(\operatorname{tr} X_{1}\right) \mathrm{Y}-\left[(1-\varepsilon) \mathrm{P}\left(\mathrm{X}_{2}\right)+\varepsilon\left(\operatorname{tr} \mathrm{X}_{2}\right) \mathrm{Y}\right]\right\|_{1} \\
& =\left\|(1-\varepsilon)\left(P\left(X_{1}\right)-P\left(X_{2}\right)\right)+\varepsilon Y-\varepsilon Y\right\|_{1}=(1-\varepsilon)\left\|P\left(X_{1}\right)-P\left(X_{2}\right)\right\|_{1} \\
& \leq(1-\varepsilon)\left\|X_{1}-X_{2}\right\|_{1} .
\end{aligned}
$$

By Lemma 2.1 (iv) we easily obtain $P_{\varepsilon} \in \mathcal{S}_{n m}$.

Combining these two results we instantly get:

Theorem 2.4. The set $\mathcal{S}_{n m}$ of all norm mixing Markov operators is a norm dense and open subset of $\mathcal{S}$.

Proof. It remains to show that $\mathcal{S}_{n m}$ is norm open. For this we notice that the set $\mathcal{S}_{n m}=\left\{P \in \mathcal{S}: \sup _{X_{1}, X_{2} \in S}\left\|P^{n}\left(X_{1}\right)-P^{n}\left(X_{2}\right)\right\|_{1}<\varepsilon\right\}$ is norm open. 
In the next theorem we study the set of mixing Markov operators with strictly positive invariant states. Let us recall:

Definition 2.5. We say that a state $X \in \mathrm{S}$ is strictly positive if for each nonzero $x \in \mathcal{H}$ we have $\langle X x, x\rangle>0$ (or equivalently that eigenvectors of $X$ span the whole space $\mathcal{H}$, or that $X$ is "1-1"). The set of all strictly positive states is denoted by $\mathrm{S}_{+}$. The set of all norm mixing Markov operators possessing a strictly positive invariant state is denoted by $\mathcal{S}_{n m+}$.

In the next result we show that the set $\mathcal{S}_{n m+}$ is still a large set in $\mathcal{S}$. Namely we have

Theorem 2.6. The set $\mathcal{S}_{n m+}$ is a dense $G_{\delta}$ subset of $\mathcal{S}$ for the norm operator topology.

Proof. Let us choose an orthonormal basis $e_{1}, e_{2}, \ldots$ in $\mathcal{H}$. For a strictly positive state $X$ we fix an orthonormal basis consisting of its eigenvectors $e_{1}^{X}, e_{2}^{X}, \ldots$ By $\pi_{l}^{X}$ we denote the orthonormal projection onto $\operatorname{lin}\left\{e_{1}^{X}, \ldots, e_{l}^{X}\right\}$. Let

$J_{k, X}=\min \left\{l:\left\|\pi_{l}^{X}(x)\right\|>1-1 / k\right.$ for all $x \in \operatorname{lin}\left\{e_{1}, e_{2}, \ldots, e_{k}\right\}$ with $\left.\|x\|=1\right\}$.

We define

$$
\varepsilon_{k}(X)=\inf \left\{\langle X x, x\rangle:\left\|\pi_{J_{k}, X}^{X}(x)\right\|>1 / 2,\|x\|=1\right\} .
$$

Note that $\varepsilon_{k}(X)>0$, as long as $X$ is strictly positive, where $k$ is arbitrary. Now let us consider an operator norm $G_{\delta}$ set

$$
\mathcal{A}=\bigcap_{N} \bigcup_{X_{N} \in \mathrm{S}_{+}} \bigcup_{n \geq N}\left\{P \in \mathcal{S}:\left\|P^{n}-Q_{X_{N}}\right\|<\frac{\varepsilon_{N}\left(X_{N}\right)}{2}\right\} .
$$

We will prove that $\mathcal{A}=\mathcal{S}_{n m+}$. The inclusion $\supseteq$ is obvious. In fact, if $P \in \mathcal{S}_{n m+}$, then for any $N \in \mathbb{N}$ it is sufficient to take $X_{N}=X_{*}$, where $X_{*}$ is a unique $P$ invariant and strictly positive state.

For the proof of the opposite inclusion $\subseteq$ we first note that $\mathcal{A} \subseteq \mathcal{S}_{n m}$. In fact, following our Lemma 2.1 it is enough to observe

$$
\begin{gathered}
\sup _{X, Y \in \mathrm{S}}\left\|P^{n}(X)-P^{n}(Y)\right\|_{1}=\sup _{X, Y \in \mathrm{S}}\left\|\left(P^{n}(X)-X_{N}\right)-\left(P^{n}(Y)-X_{N}\right)\right\|_{1} \\
=\sup _{X, Y \in \mathrm{S}}\left\|\left(P^{n}(X)-Q_{X_{N}}(X)\right)-\left(P^{n}(Y)-Q_{X_{N}}(Y)\right)\right\|_{1} \leq 2 \frac{\varepsilon_{N}\left(X_{N}\right)}{2} \rightarrow 0 .
\end{gathered}
$$

Let $X_{*}=\lim _{n \rightarrow \infty} P^{n}(X)$ be a $P$-invariant state. Suppose that, on the contrary, there exists a normalized $\psi \in \mathcal{H}$ such that $X_{*} \psi=0$. It follows from our definition of $J_{k, X}$ that

$$
\lim _{N \rightarrow \infty}\left\|\pi_{J_{N, X_{N}}}^{X_{N}}(\psi)\right\|=1
$$

Hence

$$
\begin{aligned}
0 & <\left\|X_{N}(\psi)\right\|=\left\|X_{*}(\psi)-X_{N}(\psi)\right\| \leq\left\|X_{*}-X_{N}\right\|_{1}=\left\|\left(P^{n}-Q_{X_{N}}\right)\left(X_{*}\right)\right\|_{1} \\
& \leq\left\|P^{n}-Q_{X_{N}}\right\|<\frac{1}{2} \inf \left\{\left\langle X_{N}(x), x\right\rangle:\|x\|=1,\left\|\pi_{J_{N}, X_{N}}^{X_{N}}(x)\right\|>1 / 2\right\} \leq \frac{1}{2}\left\|X_{N}(\psi)\right\|
\end{aligned}
$$

when $N$ is large enough, which is a contradiction. It follows that $X_{*}$ is strictly positive. 


\section{Category for the strong operator topology}

In this section we consider strong operator topology mixing. In comparison with the norm topology, here Markov operators with iterates converging to onedimensional projections form a meager set. We start with

Definition 3.1. We say that a Markov operator $P$ on $\mathcal{S}$ is almost mixing in the strong operator topology if for each pair of states $X_{1}, X_{2} \in \mathrm{S}$ we have

$$
\lim _{n \rightarrow \infty}\left\|P^{n}\left(X_{1}\right)-P^{n}\left(X_{2}\right)\right\|_{1}=0 .
$$

The set of all almost mixing operators is denoted by $\mathcal{S}_{\text {sam }}$. If moreover there exists $X_{*} \in \mathrm{S}$ such that for all $X_{1} \in \mathrm{S}$ we have

$$
\lim _{n \rightarrow \infty}\left\|P^{n}\left(X_{1}\right)-X_{*}\right\|_{1}=0,
$$

then the operator $P$ is called strong operator topology mixing. The set of all s.o.t. mixing Markov operators is denoted by $\mathcal{S}_{s m}$.

Clearly $\mathcal{S}_{n m} \subset \mathcal{S}_{\text {sm }} \subset \mathcal{S}_{\text {sam }}$. Since the operator norm topology is stronger than the strong operator topology, thus we easily get the following.

Lemma 3.2. The set $\mathcal{S}_{\text {sam }}$ is a strong operator topology dense $G_{\delta}$ subset of $\mathcal{S}$.

Proof. It remains to prove that $\mathcal{S}_{\text {sam }}$ is an s.o.t. $G_{\delta}$. For this recall that whenever $\mathcal{H}$ is separable (has countable orthonormal basis), then $\mathcal{C}_{1}$ is separable too (finitedimensional operators are $\|\cdot\|_{1}$ dense in $\mathcal{C}_{1}$; see [17] or [20] for all the details). Choose $Y_{1}, Y_{2}, \ldots$ a countable dense family of states in $\mathrm{S}$. It follows from the contraction argument that

$$
\lim _{n \rightarrow \infty}\left\|P^{n}\left(X_{1}\right)-P^{n}\left(X_{2}\right)\right\|_{1}=0
$$

holds for every pair of $X_{1}, X_{2} \in \mathrm{S}$ if and only if

$$
\lim _{n \rightarrow \infty}\left\|P^{n}\left(Y_{i}\right)-P^{n}\left(Y_{j}\right)\right\|_{1}=0
$$

for any pair $Y_{i}, Y_{j}$. Again, since all $P^{n}$ are contractions, thus the above convergence holds if and only if it holds for some subsequence. To end the proof it is enough to write

$$
\mathcal{S}_{\text {sam }}=\bigcap_{i, j} \bigcap_{k} \bigcap_{N} \bigcup_{n \geq N}\left\{P \in \mathcal{S}:\left\|P^{n}\left(Y_{i}\right)-P^{n}\left(Y_{j}\right)\right\|_{1}<\frac{1}{k}\right\} .
$$

The next two results show that for most of the operators in $\mathcal{S}_{\text {sam }}$ their iterates do not converge (in the $\mathrm{w}^{*}$.o.t.) to a Markov operator.

Lemma 3.3. The set $\mathcal{S}_{0}=\left\{P \in \mathcal{S}: P^{n} \rightarrow 0\right.$ in the weak* operator topology $\}$ is a strong operator topology dense subset of $\mathcal{S}$.

Proof. Let us consider an orthonormal basis $e_{1}, e_{2}, e_{3}, \ldots$ in $\mathcal{H}$. Set $E_{N}$ to be the orthogonal projection onto $\operatorname{lin}\left\{e_{1}, \ldots, e_{N}\right\}$. Given $Y_{1}, Y_{2}, \ldots, Y_{s} \in \mathrm{S}$ and fixed $P \in \mathcal{S}$ we denote by

$$
\mathcal{U}=\left\{\widetilde{P} \in \mathcal{S}: \forall_{1 \leq j \leq s}\left\|P\left(Y_{j}\right)-\widetilde{P}\left(Y_{j}\right)\right\|_{1}<\varepsilon\right\}
$$

an s.o.t. open neighborhood of $P$. We will find $\widetilde{P} \in \mathcal{U}$ such that $\widetilde{P}^{n} \rightarrow 0$ in the weak* operator topology. Let us choose $N \geq 1$ large enough and $\varepsilon^{\prime}$ small enough so 
that $\left\|Y_{j}-E_{N} Y_{j} E_{N}\right\|_{1}<\varepsilon^{\prime}$, for all $j=1, \ldots, s$ and such that the Markov operator defined below belongs to $\mathcal{U}$. Namely, let us define

$$
\begin{aligned}
& \widetilde{P}(X)=\left(1-\frac{\varepsilon}{3}\right) E_{N} P\left(E_{N} X E_{N}\right) E_{N} \\
& +\left(1-\frac{\varepsilon}{3}\right) \operatorname{tr}\left(\left(I-E_{N}\right) P\left(E_{N} X E_{N}\right)\left(I-E_{N}\right)\right)\left\langle\cdot, e_{N+1}\right\rangle e_{N+1} \\
& +\frac{\varepsilon}{3} \operatorname{tr}\left(P\left(E_{N} X E_{N}\right)\right)\left\langle\cdot, e_{N+1}\right\rangle e_{N+1}+\sigma\left(I-E_{N}\right) X\left(I-E_{N}\right) \sigma^{*},
\end{aligned}
$$

where $\sigma: \mathcal{H} \rightarrow \mathcal{H}$ is given by $\sigma\left(e_{j}\right)=e_{j+1}$ and $\sigma^{*}= \begin{cases}0, & j=1 \\ e_{j-1}, & j>1\end{cases}$

Denote $\alpha(X)=\operatorname{tr}\left(\left(I-E_{N}\right) X\left(I-E_{N}\right)\right)$, for $X \in \mathrm{S}$. We have

$$
\begin{aligned}
1 \geq \alpha & \left(\widetilde{P}^{n+1}(X)\right)=\left(1-\frac{\varepsilon}{3}\right) \operatorname{tr}\left(\left(I-E_{N}\right) E_{N} P\left(E_{N} \widetilde{P}^{n}(X) E_{N}\right) E_{N}\left(I-E_{N}\right)\right) \\
& +\left(1-\frac{\varepsilon}{3}\right) \operatorname{tr}\left(\left(I-E_{N}\right) P\left(E_{N} \widetilde{P}^{n}(X) E_{N}\right)\left(I-E_{N}\right)\right) \cdot 1 \\
& +\frac{\varepsilon}{3} \operatorname{tr}\left[P\left(E_{N} \widetilde{P}^{n}(X) E_{N}\right)\right] \cdot 1 \\
& +\operatorname{tr}\left[\left(I-E_{N}\right) \sigma\left(I-E_{N}\right) \widetilde{P}^{n}(X)\left(I-E_{N}\right) \sigma^{*}\left(I-E_{N}\right)\right] \\
& \geq \frac{\varepsilon}{3} \operatorname{tr}\left(E_{N} \widetilde{P}^{n}(X) E_{N}\right)+\alpha\left(\widetilde{P}^{n}(X)\right) \\
& =\frac{\varepsilon}{3}\left[1-\operatorname{tr}\left(\left(I-E_{N}\right) \widetilde{P}^{n}(X)\left(I-E_{N}\right)\right)\right]+\alpha\left(\widetilde{P}^{n}(X)\right) \\
& =\frac{\varepsilon}{3}\left(1-\alpha\left(\widetilde{P}^{n}(X)\right)\right)+\alpha\left(\widetilde{P}^{n}(X)\right)=\frac{\varepsilon}{3}+\left(1-\frac{\varepsilon}{3}\right) \alpha\left(\widetilde{P}^{n}(X)\right) .
\end{aligned}
$$

Now by an elementary argument the sequence $\alpha\left(\widetilde{P}^{n}(X)\right) \rightarrow 1$ if $n \rightarrow \infty$. We introduce $\beta_{i, k, n}=\left\langle\widetilde{P}^{n}(X) e_{i}, e_{k}\right\rangle$, where $i, k \geq 1$. The next three properties easily follow from our definitions of $\widetilde{P}$ and the coefficient $\alpha$. The fourth case is discussed in full detail.

Case 1. For $1 \leq i, k \leq N$ we have $\lim _{n \rightarrow \infty} \beta_{i, k, n} \rightarrow 0$.

Case 2. For $1 \leq i \leq N, k>N, n \geq 1$ we have $\beta_{i, k, n}=0$.

Case 3. For $1 \leq k \leq N, i>N, n \geq 1$ we have $\beta_{i, k, n}=0$.

Case 4. If $k, i>N$ we start with

$$
\begin{aligned}
& \left\langle\widetilde{P}^{n}(X) e_{i}, e_{k}\right\rangle=\left\langle\left(1-\frac{\varepsilon}{3}\right) E_{N} P\left(E_{N} \widetilde{P}^{n-1}(X) E_{N}\right) E_{N} e_{i}, e_{k}\right\rangle \\
& +\left(1-\frac{\varepsilon}{3}\right) \operatorname{tr}\left(\left(I-E_{N}\right) P\left(E_{N} \widetilde{P}^{n-1}(X) E_{N}\right)\left(I-E_{N}\right)\right)\left\langle e_{i}, e_{N+1}\right\rangle\left\langle e_{N+1}, e_{k}\right\rangle \\
& +\frac{\varepsilon}{3} \operatorname{tr}\left(P\left(E_{N} \widetilde{P}^{n-1}(X) E_{N}\right)\right)\left\langle e_{i}, e_{N+1}\right\rangle\left\langle e_{N+1}, e_{k}\right\rangle \\
& +\left\langle\sigma\left(I-E_{N}\right) \widetilde{P}^{n-1}(X)\left(I-E_{N}\right) \sigma^{*} e_{i}, e_{k}\right\rangle .
\end{aligned}
$$

It follows from cases $1-3$ that in the above sum the first three terms tend to 0 . It remains to show that

$$
\left\langle\sigma\left(I-E_{N}\right) \widetilde{P}^{n-1}(X)\left(I-E_{N}\right) \sigma^{*} e_{i}, e_{k}\right\rangle \rightarrow 0 \text { when } n \rightarrow \infty .
$$

For this,

$$
\begin{aligned}
& \left\langle\sigma\left(I-E_{N}\right) \widetilde{P}^{n-1}(X)\left(I-E_{N}\right) \sigma^{*} e_{i}, e_{k}\right\rangle \\
& =\left\langle\widetilde{P}^{n-1}(X)\left(I-E_{N}\right) e_{i-1},\left(I-E_{N}\right) e_{k-1}\right\rangle \\
& =\left\langle\widetilde{P}^{n-1}(X) e_{i-1}, e_{k-1}\right\rangle=\beta_{i-1, k-1, n-1} .
\end{aligned}
$$


Iterating this procedure we obtain

$$
\left\langle\widetilde{P}^{n}(X) e_{i}, e_{k}\right\rangle=\beta_{i-l, k-l, n-l}+\gamma_{n, l},
$$

where $\lim _{n \rightarrow \infty} \gamma_{n, l}=0$ for each $l \geq 1$. We have already noticed that

$$
\lim _{n \rightarrow \infty} \beta_{i-l, k-l, n-l}=0, \quad \text { whenever } l \geq(i-N) \vee(k-N) .
$$

The proof is completed.

Lemma 3.4. Let $e_{1}, e_{2}, e_{3}, \ldots$ be a fixed orthonormal basis and $Y_{1}, Y_{2}, \ldots$ a countable and dense (with respect to the norm $\|\cdot\|_{1}$ ) family of states. Then the set

$$
\bigcap_{k} \bigcap_{N} \bigcup_{n \geq N}\left\{P \in \mathcal{S}: \sum_{j=1}^{N}\left\langle P^{n}\left(Y_{k}\right) e_{j}, e_{j}\right\rangle<\frac{1}{N}\right\}
$$

is a $w^{*}$.o.t. $G_{\delta}$ and an s.o.t. dense subset of $\mathcal{S}$.

Proof. It follows directly from its representation that the above set is a w*.o.t. (hence s.o.t.) $G_{\delta}$ subset of $\mathcal{S}$. By Lemma 3.3 it is dense in the strong operator topology.

Clearly the Markov operators from the above lemma do not enjoy invariant states. The final results of our paper show that the s.o.t. and norm topologies on $\mathcal{S}$ are very different from the perspective of Baire category. Markov operators with convergent iterates are residual for the norm topology but for the s.o.t. they are rare. Using terminology from the commutative ergodic theory we may say that "sweeping" Markov operators are generic for the s.o.t. Combining the last three lemmas we obtain:

Theorem 3.5. The set

$$
\left\{P \in \mathcal{S}_{\text {sam }}: \exists_{n_{j} / \infty} \lim _{j \rightarrow \infty} P^{n_{j}}=0 \text { in } w^{*} \text {.o.t. }\right\}
$$

is a strong operator topology dense $G_{\delta}$ subset of $\mathcal{S}$.

Proof. By Lemma 3.3 the above set is an s.o.t. dense subset of $\mathcal{S}$. Lemma 3.4 guarantees that it is s.o.t. $G_{\delta}$.

We end the paper with

Theorem 3.6. The set $\left\{P \in \mathcal{S}_{\text {sam }}: P\right.$ has no invariant state $\}=\mathcal{S}_{\text {sam }} \backslash \mathcal{S}_{\text {sm }}$ is a strong operator topology dense $G_{\delta}$ subset of $\mathcal{S}$.

Proof. It is obvious that an almost mixing (in the s.o.t.) Markov operator $P$ has no invariant states if and only if $P$ is not mixing. Since (by Lemma 3.2) $\mathcal{S}_{\text {sam }}$ is strong operator topology dense $G_{\delta}$, thus the result follows from

$$
\mathcal{S}_{\text {sam }} \backslash \mathcal{S}_{\text {sm }}=\mathcal{S}_{\text {sam }} \cap \bigcap_{k} \bigcap_{N} \bigcup_{n \geq N}\left\{P \in \mathcal{S}: \sum_{j=1}^{N}\left\langle P^{n}\left(Y_{k}\right) e_{j}, e_{j}\right\rangle<\frac{1}{N}\right\},
$$

where $e_{1}, e_{2}, \ldots$ and $Y_{1}, Y_{2}, \ldots$ are the same as in Lemma 3.4. 


\section{REFERENCES}

[1] Sh.A. Ayupov and T.A. Sarymsakov, Markov operators on quantum probability spaces, Probability theory and applications, Proc. World Congr. Bernoulli Soc., Tashkent/USSR 1986, vol. 1 (1987), 445-454. MR.1092383

[2] W. Bartoszek, Norm residuality of ergodic operators, Bull. Pol. Ac. Sci. Math. vol. 29.3-4 (1981), 165-167. MR0638759 (83a:47009)

[3] W. Bartoszek, Asymptotic properties of the iterates of stochastic operators on (AL) Banach lattices, Ann. Polon. Math. 52 (1990), 165-173. MR.1087364 (92a:47007)

[4] W. Bartoszek, One-parameter positive contraction semigroups are convergent, Univ. Iagel. Acta Math. vol. 33 (1996), 49-57. MR1422437|(98d:47075)

[5] W. Bartoszek, On the residuality of mixing by convolutions probabilities, Israel J. of Math. vol. 80 (1992), 183-193. MR.1248934 (95b:22014)

[6] O. Bratteli and D.W. Robinson, Operator Algebras and Quantum Statistical Mechanics 1, Springer Verlag, 1979. MR0545651 (81a:46070)

[7] J.R. Choksi, S.J. Eigen, and V.S. Prasad, In general a stochastic operator does not preserve a measure, Indiana Univ. Math. J. vol. 36 (1987), 617-630. MR0905613 (89d:47014)

[8] J.R. Choksi and V.S. Prasad, Approximation and Baire category theorems in ergodic theory, in Proc. Sherbrooke Workshop Measure Theory (1982), Springer Lecture Notes 1033, 94-113. MR $0729529(85 \mathrm{~d}: 28011)$

[9] E.Yu. Emel'yanov and M.P.H. Wolff, Positive operators on Banach spaces ordered by strongly normal cones. Positivity and its applications (Nijmegan, 2001). Positivity 7 (2003), no. 1-2, 3-22. MR2028362

[10] E. Yu Emel'yanov and M. Wolff, Asymptotic properties of Markov semigroups on preduals of von Neumann algebras, preprint (2001).

[11] P.R. Halmos, Lectures on ergodic theory, Chelsea, New York, 1958. MR0111817 (22:2677)

[12] A. Iwanik, Approximation theorems for stochastic operators, Indiana Univ. Math. J. vol. 29 No. 3 (1980), 415-425. MF 0570690 (84m:47039a)

[13] A. Iwanik, Baire category of mixing for stochastic operators, Rend. Circ. Mat. Palermo, Serie II-28 (1992), 201-217. MR1183052 (93j:47011)

[14] A. Lasota and J. Myjak, Generic properties of stochastic semigroups, Bull. Pol. Ac. Sci. Math. vol. 40.4 (1992), 283-292. MR1402315 (97h:47038)

[15] M. Reed and B. Simon, Methods of Modern Mathematical Physics, vol. 1, Functional Analysis, Academic Press, 1972. MR0751959 (85e:46002)

[16] J.R. Retherford, Hilbert Space: Compact Operators and the Trace Theorem, London Mathematical Society Student Texts 27, 1993. MR.1237405 (95g:47001)

[17] J.R. Ringrose, Compact non-selfadjoint operators, Van Nostrand, 1971.

[18] U. Sachdeva, On category of mixing in infinite measure spaces, Math. Systems Theory vol. 5 (1971), 319-330. MR0306459 (46:5585)

[19] T.A. Sarymsakov and N.P. Zimakov, Ergodic principle for the Markov semigroup in ordered normed spaces with basis (in Russian), Dol. Akad. Nauk SSSR vol. 289 (3) (1986), 554-558. MR 0856476 (88c:47085)

[20] B. Simon, Trace Ideals and their Applications, Cambridge Univ. Press, 1979. MR0541149 (80k:47048)

Department of Mathematics, Gdańsk University of Technology, ul. Narutowicza 11/12, 80952 Gdańsk, Poland

Department of Mathematics, Gdańsk University of Technology, ul. Narutowicza 11/12, 80952 Gdańsk, Poland 